\title{
ESTRATÉGIAS PARA O ENSINO DOS VALORES EM AULAS DE EDUCACÃO FÍSICA ${ }^{1}$
}

\author{
Guy Ginciene \\ Universidade Estadual Paulista, Rio Claro, São Paulo, Brasil \\ Sara Quenzer Matthiesen \\ Universidade Estadual Paulista, Rio Claro, São Paulo, Brasil
}

\begin{abstract}
Resumo
Dada a importância do ensino dos valores na escola, esta pesquisa teve como objetivo investigar, por meio da pesquisa-ação, formas de fazê-lo, sistematizando algumas estratégias passíveis de utilização em aulas de Educação Física. Os resultados apontaram três tipos de estratégias: avaliações diárias para a discussão do comportamento dos alunos; Sport Education para o tratamento dos valores próprios da Educação Física; discussão de valores a partir de vídeos. Essas estratégias se mostraram essenciais para que os valores apareçam de uma forma sistematizada em aulas e não como fruto do acaso, em especial, quando se tem como objetivo formar o cidadão.
\end{abstract}

Palavras-chave: Valores. Educação Física. Sport Education. Pedagogia do Esporte.

\section{Introdução}

São muitos os benefícios proporcionados pelas práticas corporais para quem as pratica. Os mais comuns, apontados pela bibliografia da área, são: físico, afetivo, social e cognitivo (BAILEY et al., 2009). É evidente, no entanto, que, para alcançá-los, estratégias específicas são necessárias, uma vez que a prática, por si só, não garante que esses benefícios sejam alcançados.

O mesmo acontece no esporte, de modo que, independentemente de onde seja ensinado, pode contribuir, entre outras coisas, para a educação de crianças e jovens (MACHADO, 2012; PAES, 2001; SANTANA, 2005). Na escola, por exemplo, é importante que o esporte e os demais conteúdos da cultura corporal de movimento sejam tratados segundo as dimensões conceituais, procedimentais e atitudinais (BRASIL, 1998).

Mas será que esses aspectos, em especial a dimensão atitudinal, são tratados em aulas de Educação Física? Será que a bibliografia da área, voltada à formação de professores, e os currículos apresentam diretrizes para o trato dos valores? E as pesquisas científicas têm esse tema como preocupação?

Cabe lembrar que a dimensão atitudinal é aquela relacionada aos valores e às atitudes. Os valores nada mais são do que "crenças, guias" e "metas gerais que nos informam sobre

\footnotetext{
${ }^{1}$ Pesquisa realizada com o apoio da FAPESP.
} 
como agir melhor na vida" (PROGRAMA DAS NAÇÕES UNIDAS PARA O DESENVOLVIMENTO, 2010), enquanto as atitudes são as ações que tomamos frente a essas "crenças".

Delimitando essa reflexão aos valores, verificamos que nem sempre os conteúdos atitudinais são programados para serem implementados de forma sistematizada durante as aulas de Educação Física. Pelo contrário, nota-se que, nas poucas vezes em que esses conteúdos aparecem, são fruto do acaso, ou seja, surgem, por exemplo, para discutir um eventual problema de comportamento decorrente da aula, como bem evidenciaram alguns autores (ACEDO; DARIDO; IMPOLCETTO, 2014; FREIRE et al., 2010; IMPOLCETTO et al., 2007; MARTINS; FREIRE, 2008).

Por sua vez, uma análise de 13 obras que dão sustentação à formação de professores de Educação Física apontou que, embora os conteúdos conceituais e atitudinais sejam tratados, são abordados de forma superficial, diferentemente dos aspectos procedimentais, que, além de priorizados, são mais detalhados (FREIRE; OLIVEIRA, 2004). Essa restrita preocupação com as dimensões atitudinais também foi constatada em documentos curriculares estaduais em relação à ginástica (CARVALHO; DARIDO; IMPOLCETTO, 2015), o que, certamente, não difere em relação aos demais componentes da cultura corporal, inclusive o esporte, tema deste artigo.

Quanto às produções científicas voltadas ao ensino dos esportes, nota-se que há uma baixa produção e preocupação com a sistematização dos valores (BARROSO; DARIDO, 2009), demonstrando o quanto são, muitas vezes, deixados em segundo plano, sem que haja a atenção e o planejamento necessários para o seu ensino.

Isso nos leva a concluir que, se os conteúdos da dimensão atitudinal - diante da sua importância para a formação do cidadão (objetivo primordial da escola) - nem sempre estão presentes nas aulas de Educação Física, na bibliografia da área, nos documentos que orientam os currículos e nas produções científicas, é porque o potencial educativo dos componentes da cultura corporal não está sendo aproveitado tanto quanto poderia ou que as estratégias das aulas não são condizentes com o objetivo da escola.

Diante disso, parece necessário que haja mais esforços e estudos que se debrucem sobre essa temática, de modo a se ter maior clareza sobre "o que" ensinar em relação aos valores em aulas de Educação Física e a "como" fazê-lo. Não por outro motivo, o objetivo desta pesquisa foi o de investigar formas de sistematizar o ensino dos valores em aulas de Educação Física, tendo como base o atletismo.

\section{Metodologia}

Para atingir o objetivo desta pesquisa de caráter qualitativo (SPARKES; SMITH, 2014; THOMAS; NELSON; SILVERMAN, 2012), foram realizadas observações participantes durante um período de intervenção junto a um professor de uma escola estadual situada na cidade de Rio Claro - SP. A turma definida pelo professor para a implementação da proposta foi a do sexto ano do Ensino Fundamental.

A pesquisa foi constituída por reuniões regulares com o professor de Educação Física e em um período de intervenção de aulas de atletismo. As reuniões foram realizadas antes e durante o período da intervenção, sendo gravadas e transcritas. Já a intervenção ocorreu em dez encontros compostos por aulas duplas, totalizando 20 aulas, que, por meio da observação participante, foram registradas em um diário de campo. $\mathrm{O}$ intuito das reuniões e da intervenção foi o de encontrar estratégias para o ensino dos valores em aulas de Educação Física.

Baseados nas reuniões e nas observações das primeiras aulas da intervenção, elaboramos uma unidade didática para as últimas 12 aulas, visando ao ensino das corridas do atletismo, baseando-nos no modelo conhecido como Sport Education (SIEDENTOP; HASTIE; MARS, 2011). 
Pautando-nos na Análise de Conteúdo (BARDIN, 2011), portanto, em suas três fases"pré-análise", "exploração do material" e "tratamento dos resultados obtidos e interpretação", procedemos com a análise dos dados, baseando-nos na "análise categorial", mais precisamente na "análise temática", daí a importância da codificação dos dados obtidos nas transcrições das reuniões e das observações provenientes do diário de campo, por meio da "unidade de registro". Considerando-se que esta pode ser codificada por meio de palavras ou temas, optamos pela escolha do tema, já que este "é geralmente utilizado como unidade de registro para estudar motivações de opiniões" (BARDIN, 2011, p. 135).

Como resultado, foram encontradas quatro categorias para a apresentação e a discussão dos resultados: problemas de comportamento; estratégias de ensino; dificuldades para a implementação e mudanças de comportamento, como veremos a seguir.

\section{Resultados}

Os resultados desta pesquisa serão apresentados e discutidos conforme as categorias definidas pela Análise de Conteúdo (BARDIN, 2011), de acordo com o que segue.

\section{Problemas de comportamento}

Sabe-se que os valores nem sempre estão presentes em aulas de Educação Física de forma planejada e sistematizada, sendo propostos pelo professor como um tema a ser desenvolvido. Quando estão, são fruto do acaso, ou seja, aparecem apenas quando um conflito surge durante a aula, embutidos no chamado "currículo oculto", que consiste em formas não planejadas de aparecimento de um conteúdo, no caso, dos valores durante as aulas de Educação Física (FREIRE et al., 2010; MARTINS; FREIRE, 2008).

Esse formato não planejado de ensino de um determinado conteúdo foi observado durante a intervenção, especialmente, durante os momentos de conflito e/ou de problemas de comportamento que apareceram durante as aulas. No primeiro dia da intervenção, logo na primeira atividade, durante a divisão dos grupos, duas meninas não foram aceitas, gerando um conflito entre os alunos. Além deste, outros problemas de comportamento surgiram nas demais aulas, tais como: brigas, brincadeiras fora de hora, dificuldades de atender a solicitações simples (como sentar em círculo), conversas excessivas durante as apresentações dos colegas e explicações do professor etc.

Os problemas de comportamento dos alunos também foram mencionados pelo professor durante as reuniões, como podemos ver nesse trecho: "eu queria fazer o conceitual e o procedimental [...] porque eu achei que eles eram educados quando eu entrei na educação, mas aí eu percebi que eles não eram".

Durante a intervenção, foi observada a presença de valores "relacionados à regulação do comportamento dos alunos, para garantia do trabalho efetivo e seguro" e "relacionados à convivência humana", assim como detectados por Freire et al (2010, p. 230). Apesar disso, sabemos que esses problemas de comportamento e de convivência não são próprios da Educação Física, sendo, muitas vezes, tratados como parte do já mencionado currículo oculto.

Diante dos intensos problemas comportamentais observados nas primeiras aulas da intervenção - que constantemente atrapalhavam o desenvolvimento das atividades -, das reflexões sobre as aulas e do trato dos valores, observamos que o modelo do Sport Education poderia ser uma estratégia em potencial.

O Sport Education é um modelo para o ensino dos esportes em aulas de Educação Física que visa simular pedagogicamente uma "temporada" dos esportes profissionais, de forma que os alunos vivenciem diferentes papéis (árbitros, técnicos, atletas, imprensa etc.), aprendendo a trabalhar em grupo. Este modelo também tem como intuito criar um clima festivo, 
motivador e inclusivo, além de tratar de forma pedagógica a competição (HASTIE et al., 2013; PEREIRA et al., 2015; SIEDENTOP, 1994; SIEDENTOP; HASTIE; MARS, 2011). Logo, este modelo serviu como base para a organização da unidade didática sobre as corridas do atletismo nas últimas 12 aulas da intervenção.

Estratégias de ensino

A fim de contribuir de forma positiva para a solução dos problemas comportamentais mencionados na categoria anterior, utilizamos uma estratégia pedagógica do Sport Education, chamada "awards", ou seja, "premiação" (HARVEY; KIRK; O'DONOVAN, 2011; SIEDENTOP; HASTIE; MARS, 2011).

Tal estratégia foi adaptada para um modelo de avaliação diária, utilizada para reconhecer e premiar a equipe com melhor comportamento durante as aulas. Num primeiro momento, ocorreu uma mudança no comportamento dos alunos quando comparado às aulas sem a utilização dessa estratégia. Entretanto, a partir do terceiro encontro após essa implementação, alguns problemas, principalmente durante as explicações e apresentações das atividades na quadra esportiva, reincidiram. Foi, então, que os itens da avaliação diária serviram como uma importante estratégia para iniciar as discussões dos eventuais problemas e conflitos emergentes, fazendo com que os alunos refletissem sobre suas atitudes. Apesar dessa contribuição, ficou a dúvida se a mudança de comportamento esteve relacionada à preocupação com a pontuação ou ao fato de terem incorporado determinados valores em suas atitudes.

Certamente, o ponto positivo dessa estratégia de avaliação diária foram as reflexões sobre o comportamento, realizadas após os conflitos e ao final de todas as aulas, durante o anúncio da pontuação. Com isso, percebemos que a proposta do Teaching Personal and Social Responsibility (TPSR) (HELLISON, 2013) pode ser uma eficiente alternativa em relação ao sistema de avaliação diária, visto que o TPSR propõe conversas, reflexões em grupo e autoavaliações sobre o comportamento, além de não exigir que o professor se preocupe em pontuar as equipes durante todas as aulas.

O fato de haver estratégias para a discussão de valores específicos pode, certamente, ajudar os professores a iniciar as discussões e debates sobre eventuais problemas de comportamento e conflitos que apareçam no decorrer das aulas, sem deixar que estes passem em branco, sem discussão, como observado em pesquisas (ACEDO, 2009; CAETANO, 2014; GUIMARÃES et al., 2001) e nesse relato do professor: "Eu sempre levava essa discussão dos valores [...]. Hoje eu já...às vezes, assim, meio que faço vista grossa às vezes".

Isso reforça a importância de planejar e sistematizar a discussão de valores para garantir que sejam abordados em aulas de Educação Física. Tanto na proposta de avaliação diária desta intervenção como na proposta de Hellison (2013), a discussão e a reflexão sobre os valores estão sempre presentes no planejamento das aulas, independente de quais conflitos apareçam.

Presentes em problemas comportamentais, os valores também podem ser discutidos a partir de situações próprias da Educação Física, como, por exemplo: o respeito aos colegas de equipe, aos adversários, aos árbitros, aos espectadores, às regras; às atitudes diante da vitória e/ou da derrota, do preconceito com as diferenças (menos habilidosos, gênero, biótipos, raças etc). É fato que, do ponto de vista dos valores, não há diferença entre o respeito ao colega na sala de aula e o respeito ao colega de equipe durante uma partida, por exemplo. No entanto, quando organizamos uma disputa de corrida durante uma aula, temos a certeza de que alguns valores vão aparecer e de que atitudes serão tomadas a partir de questionamentos como: "como vou me portar quando ganhar?", "como vou me relacionar com o colega que perdeu/ganhou?", “como vou me relacionar com o árbitro?", "como vou agir diante das regras da disputa?". Sendo assim, ao planejar uma disputa de corrida, de saltos, de arremesso, de lan- 
çamentos ou um jogo, tais reflexões sobre as atitudes que teremos em face daquela situação vão aparecer. Esta, portanto, é uma forma de relacionar os valores e atitudes às situações próprias da Educação Física, como bem demonstrou Matthiesen (2014), aos sistematizá-las em relação ao atletismo.

Nesse sentido, utilizamos como estratégia na intervenção a inserção de situações do atletismo em que os alunos se deparassem com os valores. Como já apresentado, a avaliação diária foi uma das estratégias que sustentou a discussão de valores relacionados ao comportamento geral dos alunos, que também poderia ser utilizada em outras disciplinas da escola. Já como estratégia para tratar os valores próprios da Educação Física, em especial do atletismo, utilizamos o modelo do Sport Education (HARVEY; KIRK; O'DONOVAN, 2011; SIEDENTOP, 1994, 2002; SIEDENTOP; HASTIE; MARS, 2011), de forma a estruturar a parte final da intervenção. A principal característica desse modelo é a recriação de componentes do esporte institucionalizado, como a "temporada", a "filiação", o "cronograma de competição", o "evento culminante", os "registros dos recordes" e a "festividade" (SIEDENTOP, 1994, 2002).

De forma adaptada, implementamos esse modelo na escola, utilizando as seguintes características: divisão dos alunos em equipes para que trabalhassem nessa estrutura durante a unidade didática, a organização de um festival, a avaliação diária, o comitê de fair-play, o código de condutas e a vivência de diferentes papéis/funções durante um festival. Mais do que isso, procuramos utilizar as quatro formas de intervir pedagogicamente com o Sport Education, listadas por Harvey, Kirk e O’Donovan (2011) - "contrato de ética", "júri esportivo", "prática e jogos modificados/condicionados" e "premiação".

$\mathrm{O}$ "contrato de ética" nada mais é do que um acordo entre alunos e professor sobre o comportamento nas aulas de Educação Física (HARVEY; KIRK; O’DONOVAN, 2011). A maior dificuldade na implementação dessa estratégia pedagógica foi a criação de códigos de conduta pelos alunos, para além dos mencionados na explicação da atividade. Um dos motivos que podem ter influenciado a condução dessa proposta foi a inexperiência dos alunos, do professor e do pesquisador em relação a essa estratégia e ao modelo como um todo. Em função disso, constatamos com essa intervenção que a mediação do professor para conduzir essa tarefa é de extrema importância.

Já o "júri esportivo” (HARVEY, KIRK e O’DONOVAN, 2011) foi implementado durante a unidade didática, demonstrando ser uma estratégia interessante, apesar de notarmos que os alunos ficaram limitados à observação de eventuais descumprimentos das regras do atletismo (não queimar a largada, não invadir a raia do adversário etc.) durante a competição e não tanto do comportamento e atitudes dos colegas (não aceitar a derrota, desrespeitar colegas, adversários e árbitros).

Em relação a "prática e jogos modificados/condicionados", Harvey, Kirk e O’Donovan (2011) apontam para a importância de modificar os jogos para garantir o equilíbrio entre as equipes durante as disputas. No caso desta pesquisa, a corrida de revezamento foi modificada para garantir que todos os alunos pudessem participar do festival, já que não notamos uma diferença significativa entre o nível das equipes.

Já a estratégia pedagógica da "premiação" foi realizada de duas maneiras: por meio da avaliação diária - apresentada anteriormente - e por meio da pontuação do código de condutas/fair-play utilizado no festival. Dessa forma, a partir do código de condutas desenvolvido pelos alunos e pontuado pelo "júri esportivo", as duas equipes foram premiadas com maiores pontuações ao final da "temporada".

Para além dessas estratégias pedagógicas, vale destacar outros pontos percebidos como vantajosos durante a implementação do Sport Education no que diz respeito à discussão de valores em aulas de Educação Física. A divisão da classe em equipes durante a unidade didática contribuiu para colocar os alunos em situações de socialização e resolução de confli- 
tos advindos da convivência nos encontros. Essa estratégia permitiu que desenvolvessem a capacidade de trabalhar em equipe, enfrentando os problemas e conflitos emergentes (ALEXANDER; TAGGART; THORPE, 1996; CARLSON; HASTIE, 1997; GRANT, 1992; WALLHEAD; O'SULLIVAN, 2005). Observamos que, durante a intervenção, surgiram reações de descontentamento por parte de alguns alunos com relação aos colegas de equipe. Esses alunos solicitaram diversas vezes ao professor que os colegas fossem trocados de equipe, alegando que não faziam nada ou só atrapalhavam. A mudança foi negada pelo professor e pelo pesquisador, que explicaram a necessidade de aprender a lidar com os problemas emergentes, pensando em estratégias para evitar conflitos. A reação desses alunos evidenciou a dificuldade de se conviver em grupo, sendo que a estratégia apresentada por eles foi a de "fugir" do problema, ao invés de enfrentá-lo por meio de uma solução.

A escolha de um capitão para cada equipe também oportunizou um aprendizado educativo, já que os alunos tiveram que aprender a liderar e a "gerenciar" sua equipe, achando estratégias adequadas para isso, o que também foi evidenciado nas pesquisas de Carlson e Hastie (1997) e Wallhead e O'Sullivan (2005) em relação à efetividade do modelo para desenvolver essas capacidades.

Outro aspecto relevante do Sport Education é a competição, já que envolve os alunos e possibilita que situações-problema e conflitos em geral, como o descumprimento das regras, apareçam para serem discutidos. Um dos exemplos dessa situação ocorreu em uma das atividades do quarto encontro, quando os alunos deveriam percorrer distâncias diferentes em tempos predeterminados pelo professor, sem a ajuda de um marcador de tempo (cronômetro, relógio etc.). Apesar disso, um dos alunos olhou para o relógio durante o exercício, possibilitando uma grande oportunidade para a discussão de valores. A ideia, portanto, não foi simplesmente chamar a atenção do aluno ou simplesmente repreendê-lo, mas problematizar e relacionar o ocorrido com outras situações do esporte e do dia a dia de todos os alunos da turma.

Observamos, ainda, que durante a unidade didática do Sport Education - ao contrário das primeiras aulas - não houve exclusão ou preconceitos em relação ao gênero, aos menos habilidosos ou aos colegas com diferentes biótipos. Isso corrobora a literatura da área, que aponta esse modelo de ensino como uma estratégia adequada para incluir e engajar todos os alunos nas atividades (ALEXANDER; TAGGART; THORPE, 1996; ENNIS, 1999; HASTIE; DE OJEDA; LUQUIN, 2011; PEREIRA et al., 2015; PILL, 2008).

Além do Sport Education, os vídeos foram utilizados como estratégia para a discussão de valores em aulas de Educação Física. A ideia foi colocar os alunos no centro do processo de ensino-aprendizagem (DYSON; GRIFFIN; HASTIE, 2004), utilizando o dilema como estratégia, numa espécie de "dramatização" (WINSTON, 1999). Nessa aula, portanto, os alunos se depararam com dilemas ao assistirem vídeos que os levaram a refletir sobre determinadas situações, como: numa corrida, deve-se ultrapassar um adversário que se machuca a poucos metros da linha de chegada ou deve-se parar para ajudá-lo? Qual atitude tomar como árbitro, organizador do evento e competidor quando ocorrer uma situação como a de Ben Johnson nos Jogos Olímpicos de Seul, em 1988, ao ser flagrado no exame antidoping? Vivenciando os diferentes papeis (árbitro, atleta, organizador), os alunos puderam, neste caso, discutir sobre o doping no esporte e as atitudes que tomariam perante essa situação, refletindo sobre valores de justiça que, certamente, estarão presentes em situações diversas ao longo de sua vida.

Dificuldades na implementação

Durante a realização da pesquisa, observamos alguns aspectos que dificultaram a implementação das estratégias para tratar valores em aulas de Educação Física, apresentadas no 
presente artigo. Uma delas se refere à relação entre o professor e as estratégias e a outra, em relação à receptividade dos alunos quanto a estas estratégias.

É fato que, para colocar em prática o "como ensinar", faz-se necessário que o professor conheça, aprenda e se aperfeiçoe na utilização de estratégias. O Sport Education, por exemplo, considerado, muitas vezes, como difícil de ser implementado, uma vez que demanda muito trabalho, planejamento e organização, confirma a necessidade de o professor se inteirar desta proposta (MCCAUGHTRY et al., 2004).

Utilizar esse modelo mais vezes, para ganhar experiência e passar por processos de formação que também possibilitem essas vivências, são estratégias sugeridas (HASTIE; DE OJEDA; LUQUIN, 2011; MCCAUGHTRY et al., 2004; MCMAHON; MACPHAIL, 2007; STRAN; CURTNER-SMITH, 2010) para aprender a utilizar o Sport Education.

Outro problema percebido durante a implementação foi em relação à dificuldade dos alunos para se adaptarem à nova abordagem, que os coloca no centro do processo de aprendizagem, exigindo mais autonomia, organização, participação, criatividade, habilidade para solucionar conflitos e trabalho em grupo.

Durante o período de intervenção, observamos que os alunos não estavam acostumados aos trabalhos em grupos. Isso foi evidenciado por conta da pouca autonomia para solucionar os conflitos que surgiam no decorrer das atividades. Quando um membro do grupo não ajudava ou discordava dos colegas, era comum que recorressem ao professor ou ao pesquisador para que resolvessem o conflito. Poucos foram os momentos de enfrentamento do problema em busca de soluções.

Apesar de esse fato ter sido um ponto de dificuldade, constatamos também a importância de realizar trabalhos como estes, que ofereçam a oportunidade aos alunos de resolverem os conflitos que comumente surgem em trabalhos em grupo. O professor, nessas situações, pode se colocar no papel de mediador, tentando fazer com que os próprios alunos solucionem esses problemas, estimulando-os a terem autonomia nessas situações.

Vale destacar que essa dificuldade foi amenizada com o passar das aulas, na medida em que se acostumaram com a abordagem. Isso reforça a necessidade do tempo para que os professores se apropriem dessas novas estratégias e os alunos se acostumem com essa lógica de aula.

Mudança de comportamento

Embora não fosse objetivo desta pesquisa avaliar cientificamente as mudanças de comportamento e atitudes dos alunos, observamos que algumas estratégias contribuíram para o bom comportamento, conforme relatado anteriormente.

Avaliar mudanças de comportamento e atitudes dos alunos depois de uma intervenção como esta é uma tarefa difícil. Dependendo da avaliação realizada, poderíamos dizer que os alunos aprenderam valores, mas isso não quer dizer que eles teriam uma mudança de atitude. Indo mais além, poderíamos colocar os alunos em situações de jogo, disputa e demais competições para detectar se os valores aprendidos foram incorporados, sendo, portanto, transferidos para outras situações. Isso também não quer dizer que os alunos levariam essas atitudes para além das aulas. Um exemplo disso é a pesquisa de Hassandra et al. (2007), que detectaram atitudes de fair-play de uma turma durante aulas de Educação Olímpica e após dois meses do término das aulas. Apesar disso, os alunos não apresentaram as mesmas atitudes quando colocados em situação de jogo com uma turma que não havia participado deste programa (HASSANDRA et al., 2007).

Apesar disso e de toda a dificuldade para avaliar e detectar mudanças efetivas no comportamento dos alunos fora da escola, o trabalho com valores ainda é de extrema importância, em especial, quando utilizadas as estratégias relatadas nesta pesquisa. Se desejamos formar 
alunos que saibam conviver em grupo, respeitar as diferenças e respeitar as normas, precisamos colocá-los para vivenciar, refletir e tentar solucionar, de forma pacífica, os problemas e conflitos que aparecem durante as aulas, como ocorreu ao longo da intervenção realizada nesta pesquisa. $\mathrm{O}$ fato de ainda não conseguirmos comprovar cientificamente que essas abordagens vão mudar o comportamento dos alunos no futuro não desmerece as estratégias abordadas. Pelo contrário, elas são fundamentais para colocar os alunos em situações parecidas com as que devem enfrentar no dia a dia.

Por isso, segundo todas as observações realizadas ao longo desta pesquisa, constatamos que essas estratégias podem ser consideradas como recursos possíveis para tratar os valores em aulas de Educação Física escolar. Além disso, recomendamos que mais pesquisas sejam feitas, tanto para verificar eventuais mudanças de comportamento numa perspectiva longitudinal (HASTIE; DE OJEDA; LUQUIN, 2011; WALLHEAD; O'SULLIVAN, 2005), como para entender as melhores formas de se colocar essas estratégias em prática.

\section{Considerações finais}

Se pretendemos ensinar valores, precisamos de estratégias condizentes com esse objetivo, pois a simples prática da Educação Física não vai, necessariamente, ensinar valores ou promover mudanças nas atitudes das crianças.

Em relação a "como" ensinar valores em aulas de Educação Física escolar, observamos nesta pesquisa algumas estratégias possíveis. Uma delas visa à discussão e à reflexão sobre o comportamento geral dos alunos durante as aulas, podendo ser utilizada em outras disciplinas, já que não depende das particularidades da Educação Física - como a disputa, os jogos, os adversários etc. - para ser implementada. Nesta pesquisa, foi utilizada a estratégia da avaliação diária, que também pode ser substituída por outros formatos, como o próprio TPSR.

Já em relação à estratégia para tratar os valores próprios da Educação Física, observamos que o Sport Education pode ser mais um recurso possível, já que este possibilita, por conta de seu formato, oportunidades para que os alunos vivenciem situações que colocam os valores em prática, como ocorreu nesta pesquisa. Se isso fará com que os alunos mudem seus comportamentos e atitudes, não saberemos, mas não é por esse motivo que devemos deixar que a aula ocorra livremente, sem uma estratégia adequada, principalmente quando nosso objetivo é formar o cidadão, como deveria acontecer nas aulas de Educação Física escolar. O ensino da Educação Física, quando não está bem direcionado e intencionado, pode levar a discussões, brigas, desrespeito etc. Por esse motivo, a intervenção e a estratégia utilizadas pelo professor são fundamentais.

Outra estratégia utilizada para tratar os valores próprios da Educação Física e que se mostrou igualmente eficaz foi a utilização de vídeos sobre a história do atletismo que retrataram dilemas passíveis de discussão e reflexão sobre os valores.

Nesta pesquisa, notamos uma dificuldade inicial, por parte dos alunos, de trabalhar em equipe e com a proposta de ensino do Sport Education, que os coloca no centro do processo de aprendizagem. Essa dificuldade foi diminuindo com o passar das aulas, à medida que os alunos se acostumaram com essa nova abordagem, já que, no ensino tradicional, não estão habituados com essa dinâmica, ou seja, estão acostumados simplesmente a receber informações e não, necessariamente, a fazer parte da construção do conhecimento.

Estratégias como as apresentadas nesta pesquisa são essenciais para que os valores apareçam de uma forma sistematizada e planejada durante as aulas de Educação Física e não como fruto do acaso. Isso se torna mais importante ainda quando se tem como objetivo formar o cidadão, como é o caso da Educação Física escolar. É preciso, no entanto, colocar isso tudo em prática. 


\title{
STRATEGIES TO TEACH VALUES IN PHYSICAL EDUCATION CLASSES
}

\begin{abstract}
With the importance of the values in school, this research had the purpose to investigate approaches to teach values in Physical Education, based on an action research. The results showed three kinds of approaches: daily evaluation to discuss student's behaviour; Sport Education to teach values of Physical Education; videos about tack and field to discuss values. These approaches showed as an important strategy for values appear on a organize structure in classes and not by random. This is more important when the aim is to build a citizen.
\end{abstract}

Keywords: Values. Physical Education. Sport Education model. Sport Pedagogy.

\section{ESTRATEgIAS PARA LA ENSEÑANZA DE VALORES EN CLASES DE EDU- CACIÓN FÍSICA}

\section{Resumen}

Dada la importancia de la enseñanza de valores en la escuela, el objetivo de este estudio fue investigar, mediante la investigación-acción, maneras de hacerlo en las clases de educación física. Los resultados mostraron tres tipos de estrategias: las evaluaciones diarias para la discusión del comportamiento de los estudiantes; Sport Education para el tratamiento de los valores específicos de la educación física; videos de situaciones del Atletismo para la discusión de valores. Estas estrategias han demostrado ser esenciales para que los valores aparecen durante la clase y no como el resultado de la casualidad, sobre todo cuando se pretende formar ciudadanos.

Palabras clave: Valores. Educación Física. Sport Education. Pedagogía del Deporte.

\section{Referências}

ACEDO, L. M.; DARIDO, S. C.; IMPOLCETTO, F. M. Valores e atitudes na produção científica da Educação Física brasileira: tendências e perspectivas. Coleção Pesquisa em Educação Física, v. 13, n. 3, p. 149-158, 2014.

\section{ACEDO, L. M. Valores e atitudes na prática pedagógica do professor de Educação}

Física. Universidade Estadual Paulista Júlio de Mesquita Filho, 2009.

ALEXANDER, K.; TAGGART, A.; THORPE, S. A Spring in their steps? Possibilities for professional renewal through Sport Education in Australian schools. Sport, Education and Society, v. 1, n. 1, p. 23-46, 1996.

BAILEY, R. et al. The educational benefits claimed for physical education and school sport: an academic review. Research Papers in Education, v. 24, n. 1, p. 1-27, 2009.

BARDIN, L. Análise de Conteúdo. Lisboa: Edições 70, 2011.

BARROSO, A. L. R.; DARIDO, S. C. A pedagogia do esporte e as dimensões dos conteúdos: conceitual, procedimental e atitudinal. Revista da Educação Física/UEM, v. 20, n. 2, p. 281 289, 2009. 
BRASIL. Parâmetros Curriculares Nacionais: terceiro e quarto ciclos do Ensino Fundamental. Ministério da Educação, 1998.

CAETANO, A. O jogo nas aulas de Educação Física e suas implicacões no desenvolvimento moral. Pensar a Prática, v. 17, n. 3, p. 783-799, 2014.

CARLSON, T.; HASTIE, P. The student social system within sport education. Journal of Teaching in Physical Education, v. 16, n. 2, p. 176-195, 1997.

CARVALHO, A. O.; DARIDO, S. C.; IMPOLCETTO, F. M. Análise do conteúdo de ginástica nos currículos estaduais brasileiros. Arquivos em Movimento, v. 11, n. 1, p. 54-73, 2015.

DYSON, B.; GRIFFIN, L. L.; HASTIE, P. Sport Education, Tactical games, and cooperative learning :theoretical and pedagogical considerations. Quest, v. 56, n. 2, p. 226-240, 2004.

ENNIS, B. C. D. Creating a culturally relevant curriculum for desengaged girls. Sport, Education and Society, v. 4, p. 31-49, 1999.

FREIRE, E. D. S. et al. A dimensão atitudinal nas aulas de Educação Física: conteúdos selecionados pelos professores. Revista da Educação Física/UEM, v. 21, n. 2, p. 223-235, 2010.

FREIRE, E. D. S.; OLIVEIRA, J. G. M. DE. Educação Física no Ensino Fundamental : identificando o conhecimento de natureza conceitual, procedimental e atitudinal. Motriz, v. 10, n. 1991, p. 141-151, 2004.

GRANT, B. C. Integrating sport Into the Physical Education curriculum in New Zealand secondary Schools. Quest, v. 44, n. 3, p. 304-316, 1992.

GUIMARÃES, A. A. et al. Educação Física escolar : atitudes e valores. Motriz Rio Claro, v. 7, p. 17-22, 2001.

HARVEY, S.; KIRK, D.; O’DONOVAN, T. M. Sport Education as a pedagogical application for ethical development in physical education and youth sport. Sport, Education and Society, n. October 2014, p. 1-22, 2011.

HASSANDRA, M. et al. A fair play intervention program in school Olympic education. European Journal of Psychology of Education, v. 22, n. 2, p. 99-114, 2007.

HASTIE, P. et al. The development of skill and knowledge during a sport education season of track and field athletics. Resarch Quarterly for Exercise and Sport, v. 84, n. 3, p. 336-44, 2013.

HASTIE, P. A.; DE OJEDA, D. M.; LUQUIN, A. C. A review of research on Sport Education: 2004 to the present. Physical Education \& Sport Pedagogy, v. 16, n. 2, p. 103-132, 2011.

HELLISON, D. Teaching personal and social responsibility through physical. [s.l.]: Human Kinetics, 2013. 
IMPOLCETTO, F. M. et al. Educação Física no Ensino Fundamental e Médio: a sistematização dos conteúdos na perspectiva de docentes universitários. Revista Mackenzie de Educação Física e Esporte, v. 6, n. 1, p. 89-109, 2007.

MACHADO, G. V. Pedagogia do Esporte: organização, sistematização, aplicação e avaliação de conteúdos esportivos na educação não formal. Campinas, SP: Universidade Estadual de Campinas, 2012.

MARTINS, C. D.; FREIRE, E. DOS S. Conteúdos atitudinais nas aulas de Educação Física : um estudo de caso. Revista Mackenzie de Educação Física e Esporte, v. 7, n. 3, p. 21-28, 2008.

MATTHIESEN, Sara Quenzer. Atletismo na escola. Maringá: Eduem, 2014.

MCCAUGHTRY, N. et al. Learning to teach Sport Education: misunderstandings, pedagogical difficulties, and resistance. European Physical Education Review, v. 10, n. 2, p. 135155, 2004.

MCMAHON, E.; MACPHAIL, A. Learning to teach sport education: the experiences of a pre-service teacher. European Physical Education Review, v. 13, n. 2, p. 229-246, 2007.

PAES, R. R. Educação Física escolar: o esporte como conteúdo pedagógico do ensino fundamental. Canoas: Editora da ULBRA, 2001.

PEREIRA, J. et al. A comparative study of students' track and field technical performance in sport education and in a direct instruction approach. Journal of Sports Science and Medicine, v. 14, n. 1, p. 118-27, 2015.

PILL, S. A teachers' perceptions of the Sport Education model as an alternative for upper primary school physical education. ACHPER Heathy Lifestyle Journal, v. 55, p. 23-29, out. 2008.

PROGRAMA DAS NAÇÕES UNIDAS PARA O DESENVOLVIMENTO. Relatório de Desenvolvimento Humano Brasil 2009/2010 - valores e desenvolvimento humano. [s.l: s.n.].

SANTANA, W. C. Pedagogia do Esporte na infância e complexidade. In: PAES, R. R.; BALBINO, H. F. (Eds.). . Pedagogia do Esporte: contextos e perspectivas. Rio de Janeiro: Guanabara Koogan, 2005.

SIEDENTOP, Daryl. Sport Education: Quality PE through positive sport experiences. [S.l: s.n.], 1994.

SIEDENTOP, D. Sport Education: a retrospective. Journal of Teaching in Physical Education, v. 21, 2002.

SIEDENTOP, D.; HASTIE, P.; MARS, H. VAN DER. Complete guide to Sport Education. 2. ed. [s.1.] Human Kinetics, 2011.

SPARKES, A. C.; SMITH, B. Qualitative research methods in sport, exercise and health. 
London: Routledge, 2014.

STRAN, M.; CURTNER-SMITH, M. Impact of different types of knowledge on two preservice teachers' ability to learn and deliver the Sport Education model. Physical Education \& Sport Pedagogy, v. 15, n. 3, p. 243-256, 2010.

THOMAS, J. R.; NELSON, J. K.; SILVERMAN, S. J. Métodos de pesquisa em atividade física. 6 ed. Porto Alegre: Artmed, 2012.

WALLHEAD, T.; O'SULLIVAN, M. Sport Education: physical education for the new millennium? Physical Education and Sport Pedagogy, v. 10, n. 2, p. 181-210, 2005.

WINSTON, J. Theorising drama as moral education. Journal of Moral Education, v. 28, n. 4, p. 459-471, 1999.

Recebido em: 07/11/2016

Revisado em: 07/11/2017

Aprovado em: 08/11/2017

Endereço para correspondência:

guy_ginciene@ hotmail.com

Guy Ginciene

Universidade Estadual Paulista Júlio de Mesquita Filho, Instituto de Biociências de Rio Claro, Departamento de Educação Física.

Av. 24A, n. 1515

Bela Vista

13506-900 - Rio Claro, SP - Brasil - Caixa-postal: 199 